\title{
DEVELOPMENT OF A WEB-BASED INDOOR NAVIGATION SYSTEM USING AN ACCELEROMETER AND GYROSCOPE: A CASE STUDY AT THE FACULTY OF NATURAL SCIENCES OF COMENIUS UNIVERSITY
}

Tomáš ŠTEFANIČKA ${ }^{1}$, Renata ĎURAČIOVÁ ${ }^{*}$, Csaba SERES ${ }^{1}$

\section{Abstract}

As a complex of buildings, the Faculty of Natural Sciences of the Comenius University in Bratislava tends to be difficult to navigate in spite of its size. An indoor navigation application could potentially save a lot of time and frustration. There are currently numerous technologies used in indoor navigation systems. Some of them focus on a high degree of precision and require significant financial investment; others provide only static information about a current location. In this paper we focused on the determination of an approximate location using inertial measurement systems available on most smartphones, i.e., a gyroscope and an accelerometer. The actual position of the device was calculated using "a walk detection method" based on a delayed lack of motion. We have developed an indoor navigation application that relies solely on open source JavaScript libraries to visualize the interior of the building and calculate the shortest path utilizing Dijsktra's routing algorithm. The application logic is located on the client side, so the software is able to work offline. Our solution represents an accessible lowcost and platform-independent web application that can significantly improve navigation at the Faculty of Natural Sciences. Although our application has been developed on a specific building complex, it could be used in other interiors as well.
Address

1 Department of Theoretical Geodesy, Faculty of Civil Engineering, Slovak University of Technology in Bratislava, Slovakia

* Corresponding author: renata.duraciova@stuba.sk

Key words

- Indoor navigation,

- Inertial measurement systems,

- Dijsktra's routing algorithm

- Web map application.

\section{INTRODUCTION}

In the early years of the $21^{\text {st }}$ century, devices such as pocket PCs were rare and had not yet been equipped with so many auxiliary hardware components such as digital cameras, a Global Positioning System (GPS) signal receiver, or motion detector. Nowadays mobile phones and tablets are an integral part of society. Every year handheld or wearable devices offer a superior performance compared to their predecessors with new gadgets, while being more affordable and accessible.

In general, navigation systems mainly emerged because of the accessibility of hand-held devices. Even though outdoor navigation systems have been used for years, the same does not strictly apply to their indoor counterparts. While outdoor navigation intended for drivers or the military can work with GPS or GLObal NAvigation Satellite System (GLONASS) systems (Groves, 2008; Hofmann-Wellenhof et al., 2001; Leick, 2004), an indoor navigation system has to utilize other techniques because of the lack of a satellite signal inside an indoor environment. Depending on many factors such as their degree of accuracy, response time or financial demands, various methods can be used to determine the actual location of a device. Until now, many technologies have been developed and used for detection of a device in interiors, e.g., multisensor-based navigation; Wireless Fidelity (Wi-Fi) triangulation (Ma et al., 2015; Shandilya and Idate, 
2015); Radio Frequency IDentification (RFID) used in conjunction with Near Field Communication (NFC) (Geng et al., 2014); ultrasound (Gualda et al, 2014); inertial systems (Woodman, 2007); etc. These technologies have been described in detail in many studies (Dardari et al., 2015; Gu et al., 2009; Jeon et al., 2015; Koyuncu and Yang, 2010; Mautz, 2009; Mautz, 2012; Ozdenizci et al., 2015; Yasir et al., 2016). They are used for different purposes. Some of the mentioned technologies are intended for machines in warehouses; others aim to achieve a simpler goal, i.e., to prevent people from getting lost

The building complex of the Faculty of Natural Sciences (FNS) at the Comenius University in Bratislava is not as large as other similarly purposed buildings. However, due to the complexity of the structure, which hosts hundreds of rooms within five blocks and with various amounts of floors, one can easily spend a lot of time just trying to find the target destination. This especially applies to newcomers and occasional visitors. On the other hand, the FNS complex is a good candidate for an indoor navigation system, thanks to its straight halls and cube-shaped blocks.

For this reason, our goal was to design and create an alternative indoor navigation system that would meet the following conditions:

1. Sufficient degree of accuracy in establishing the location

2. Low financial and time investments to implement a solution

3. Accessibility and platform independence

4. Usage without the need for a network connection

Based on these conditions, we have developed a simple webbased, mobile-friendly, and offline-capable map application.

Every navigation system should at least visualize a map with the current position highlighted and the best path to the specified destination; hence this paper is structured as follows: Section 2 provides a brief overview of the technologies generally used to determine the position inside of an interior as well as a justification of the technology and methods we adopted. Section 3 discusses every aspect of the implementation of the application: an overview of the architecture and the technologies used to develop our application, an analysis of the available data sources, the routing, position calculations, and visualizing the map. The results and discussion are presented in Section 4. The conclusions derived from the proposed solution are briefly summarized in Section 5.

\section{DETERMINATION OF A POSITION IN AN INDOOR ENVIRONMENT}

As mentioned before, there are many technologies that can be used to determine a device's actual location. Some of them require the establishment of an infrastructure, i.e., a network of signal emitters. Others are much more passive and provide only static information about the location. Furthermore, some can also be used with multiple mathematical methods to calculate the position from measurements. All of them have their advantages as well as disadvantages.

\subsection{Overview of Technologies}

The RFID and the NFC are two quite similar wireless communication technologies. Both employ radio signals for various kinds of tagging and tracking purposes. RFID uses electromagnetic fields in a radio frequency range to transfer data from a tag attached to an object for the purpose of its automatic identification and tracking. The estimation of the RFID position is based on electromagnetic communication between the RFID reader and the RFID tag (Rodriguez et al., 2014). RFID tags can be either active (relying on a local power source) or passive (using energy from a nearby RFID reader). Hence, active tags possess the ability to broadcast their signal over a much longer distance, while passive tags are limited to the range of the receiver. Indoor tracking with RFID systems is described, for example, in (Geng, et al., 2014). NFC is a bidirectional short-range high frequency wireless communication technology that enables devices to establish communication between themselves. It is usually limited to a close proximity, which is rarely larger than a few centimeters (Choo et al., 2014). NFC operates on the same frequency as high frequency RFID readers and may act as both a reader and a tag. An NFC-based indoor navigation system enables navigation inside of a building using frequent location updates near fixed receivers positioned in the interior (Ozendici et al., 2015).

Ultrasonic (or ultrasound) location systems rely on the measurement of an ultrasonic signal and the time of its arrival, which is calculated from the velocity of a sound received from an emitter (Ijaz et al., 2013). The location of the mobile nodes (usually tags placed on robots or worn by persons) is obtained via multilateration based on measurements of distance to static nodes permanently mounted on walls or ceilings. Multilateration is a method based on the difference in the distance between at least two emitters that broadcast their signals at known times from known static positions (Mautz, 2012). Ultrasonic location systems for improved indoor positioning are described in depth in (Hazas and Hopper, 2006), and indoor navigation using an ultrasonic sensor is presented in (Lim et al., 2014).

Wi-Fi or Wireless Local Area Networks (WLAN) triangulation can be used to determine the location of a device within a Wi-Fi network's range (Mautz, 2012). Calculating a position via triangulation relies on the geometric properties of triangles. Hence, the Wi-Fi triangulation method depends on the connections to the Wi-Fi routers. The device receives a signal emitted from a router while measuring its properties, i.e., the time of its arrival, the angle of the arrival, and the strength of the signal received. Those properties are used to estimate the angles and distances to the known position of the signal emitters and calculate their approximate position. Triangulation requires at least three signal emitters, but generally the more emitters in the range of the device, the more accurate the estimation (Ma et al., 2015).

The Quick Response (QR) code is a type of matrix barcode that is used to provide easy access to information through a device equipped with a camera. It can be scanned and then processed with software that converts the matrix cells within the code into a string of characters. In the case of the indoor navigation, QR codes are designed to provide the current location of the object where the code is placed. The most common application of QR codes within the context of indoor navigation is their distribution throughout a building near points of interest (POI) or intersecting points of paths. They can provide both a current location as well as additional useful information (Costa-Montenegro et al., 2011).

Inertial Measurement Systems (IMS) or Inertial Navigation Systems generally incorporate data from motion and rotation sensors to calculate a location (Jain et al., 2013). An orientation sensor (gyroscope) measures the angular velocity of the device. The motion sensor (accelerometer) measures the linear acceleration from which it is possible to determine the distance the device has moved. If both the device's orientation and motion are known at the time, they can be used to calculate the position relative to the initial position, i.e, the motion vector. Hence, if the previous position is known, the current position can be updated by the motion vector, which represents the distance between the origin, the destination and its azimuth.

While the gyroscope provides measurements that can be used directly (the azimuth), the distance has to be computed. There are several methods that can be used to calculate the trajectory of the device using the IMS (Le et al., 2009):

1. double integration of the acceleration

2. step detection

3. walk detection 
In theory, the double integration of acceleration is the most exact method. The velocity can be calculated by integrating the inertial acceleration over time and then deducing the position relative to the origin. However, Woodman (2007) has proved that this technique provides accurate data only initially. With each measurement, there is a certain deviation from the actual position that stacks with the previous one. Hence, deviations increase quadratically over the observation time, which is unacceptable for longer routes.

According to Ilkovičová et al. (2016), the detection of footsteps with motion sensors yields significant results. Those can be achieved by employing a variety of data processing algorithms and filters to detect the footsteps and their length. There are two disadvantages of this approach. Firstly, there is a certain risk of "the detection of false steps" that can decrease the degree of accuracy. Secondly, to estimate the accurate distance with each step, it is necessary to use an algorithm that is able to "learn the length of a person's footstep". Otherwise, a constant step length, such as an average or median step, has to be assumed (Woodman, 2007).

"Walk detection", sometimes also referred as "motion detection", is the simplest method based on the principle of measuring walking time. Distance can be calculated from the measured walk time and walk speed. Similar to the detection of footsteps, this technique requires the known motion speed of an individual, either by utilizing some kind of machine learning algorithm or assuming a constant speed.

\subsection{Applicability of Technologies in Web-Based Software}

To create a web-based application without a need for the installation of the components from third parties, two constraints have to be met: the technology must be available in hand-held devices and must provide a JavaScript Application Programing Interface (API) browser. However, there are other factors that have to be considered as well: the general accessibility of the technology, degree of precision provided, or the financial investments required.

The primary disadvantage of the RFID and ultrasonic technologies are their core requirements: signal transmitters and readers. While the cost and efficiency of such hardware tends to be variable, tags are required to be carried on the device that is being tracked. This fact violates our principle of an easily accessible application. What is more, at the time of writing this paper, there was no browser with API for the NFC or ultrasound technologies. Hence we could not adapt them for our navigation system.

Wi-Fi triangulation yields exceptional results, but larger buildings such as FNS tend to lack the required amount of Wi-Fi routers, thus rendering this technology as not entirely reliable. Furthermore, at the time of the development of the application (October 2016-January 2017), there was no browser with implemented API that could provide the capabilities required for Wi-Fi signal processing.

QR codes do not really provide a means for the dynamic location of a device; instead it just provides static information about the specific point of interest (POI). This approach also requires time and a financial investment to mark the POIs in the interior. Therefore, we decided not to use this technology. However, QR codes have potential as a supplementary means to determine a user's original location and additional information about a specific place.

The main disadvantage of an IMS is its lack of precision. Deviations have to be expected, even for a high-quality accelerometer and gyroscope. On the contrary, the device's motion and orientation sensors are standard equipment for the majority of smartphones and tablets. Using an IMS does not require any investment for the development or maintenance of any kind of infrastructure such as ultra- sound emitters. Nor does it need any additional hardware to be carried by a user as RFID does. Furthermore, unlike the QR codes, IMS is capable of dynamic navigation with the cross-browser support of the JavaScript API. For the reasons above, we chose the IMS as our primary technology for the determination of a position.

As for our techniques, we decided not to use the double integration of the acceleration because of its low degree of accuracy. The building complex of FNS is too large to rely on a method, the precision of which dramatically decreases over a longer period of time. On the other hand, short distances are quite suitable, and it could be appropriate to detect changes in floors while using an elevator or staircase. As to footstep and walk detection, both rely on unknown variables (the length of a step or the speed of a walk, respectively) that have to be set for each individual or assumed as a constant. Hence the accuracy of both methods directly depends on the individual and can more or less vary among people. However, walk detection provides a simpler and easy-to-implement solution without any risk of "false step" detection. It has also proved to be sufficient enough to navigate inside FNS; thus, we chose this approach for our navigational system.

\section{DESIGN AND IMPLEMENTATION}

The primary objective was to create both an efficient and lowcost software application for indoor navigation for FNS. Since our secondary goal was also to create easily accessible software, we preferred a web platform. Web applications can basically be launched by using any browser that supports the required APIs. Browsers are almost independent of an operating system or a device's screen size and resolution; therefore, the web platform allowed us to tailor our indoor navigation to the needs and capabilities of FNS.

\subsection{Overview of the Architecture}

While it is possible to take advantage of technologies such as Adobe's Flash, Oracle's Java or Microsoft's Silverlight to develop indoor navigation software, we decided not to use those tools, because they require the user to install third party components. Additionally, the client side of web applications is largely created with a mixture of Hypertext Markup Language (HTML), Cascading Style Sheets (CSS) and JavaScript.

As mentioned before, the application has to visualize a map with the route calculated and the user's actual location. For this purpose we adopted OpenLayers (OL) v. 3.14.0. OL is an open source JavaScript library released under a 2-clause BSD License (http://openlayers.org). Its main purpose is to load and display various types of spatial data on a web page in the form of maps. OL enables the fast and fluent rendering of both high-DPI scans and vector geometries, mainly because of its canvas - rendering system. It also implements an ability to rotate a map at any angle, which is crucial for indoor navigation software. While the OL library fully satisfied our needs, it is worth noting that there are other similar-purpose libraries, such as the open source Leaflet library (http://leafletjs.com/) or the proprietary ESRI ArcGIS API for JavaScript (https://developers.arcgis.com/javascript/).

Since minimization of the network traffic promotes the low-cost and accessibility of the solution, we therefore decided to shift the calculations of the shortest path from the server to the client. The jKstra graph and routing JavaScript library perfectly suits this need because of its lightweight design. It exposes an API for graph manipulation as well as the shortest path algorithms of Dijkstra (Dijkstra, 1959) and $\mathrm{A}^{*}$ (Zheng and Church, 2009). It is also available under an MIT license as the Git repository from the GitHub (https://github.com/ bbecquet/jKstra). Although we are aware of other routing algorithms 
and JavaScript graph libraries, the jKstra library provides sufficient calculating speed and a developer-friendly API, so we decided to use it in our application.

As a back-end technology, we chose Node.js (https://nodejs.org) v. 4.4.7, mainly for its performance, stability and ability to run on all major platforms. To keep the hardware requirements to the minimum, we adopted Express (http://expressjs.com/) as our web server frame- work. It serves application source codes (HTML, CSS and JavaScript files) as well as emergency evacuation plan scans and GeoJSON files containing the vector data required to construct a graph. Naturally, the communication between the web server (Node.js) and the client (web browser) takes place over the Hypertext Transfer Protocol (HTTP). The system's architecture, its components and their relations are represented in Figure 1.

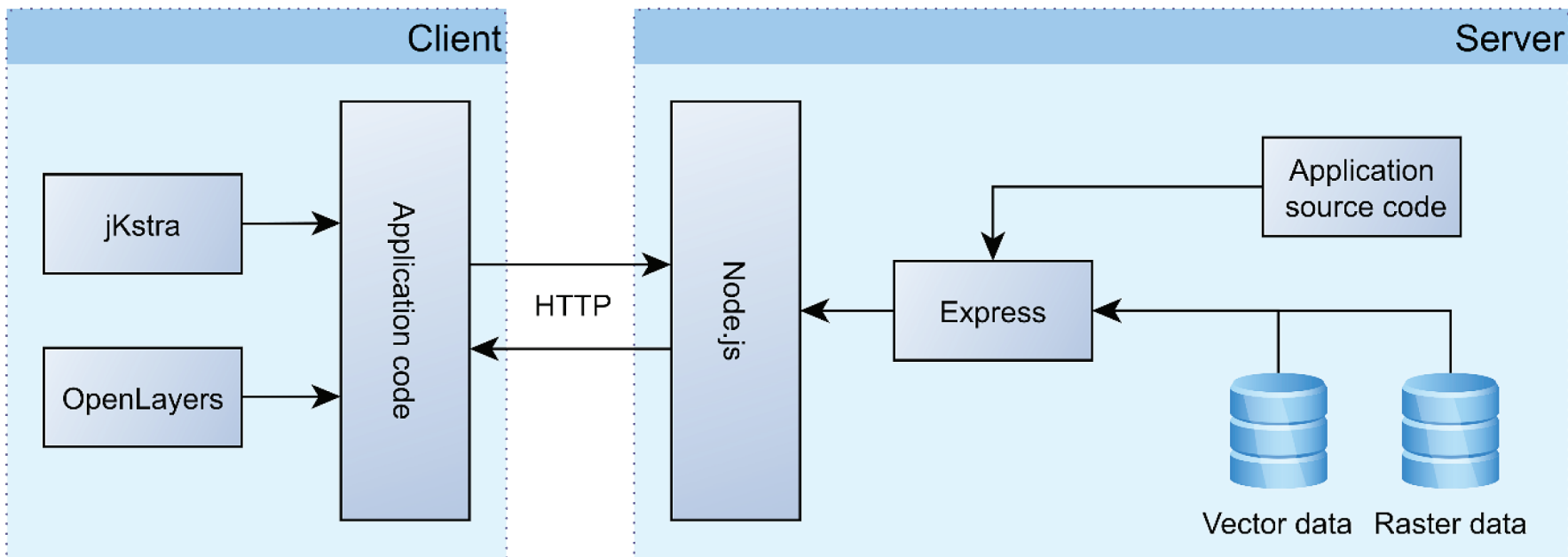

Fig. 1. Architecture of application

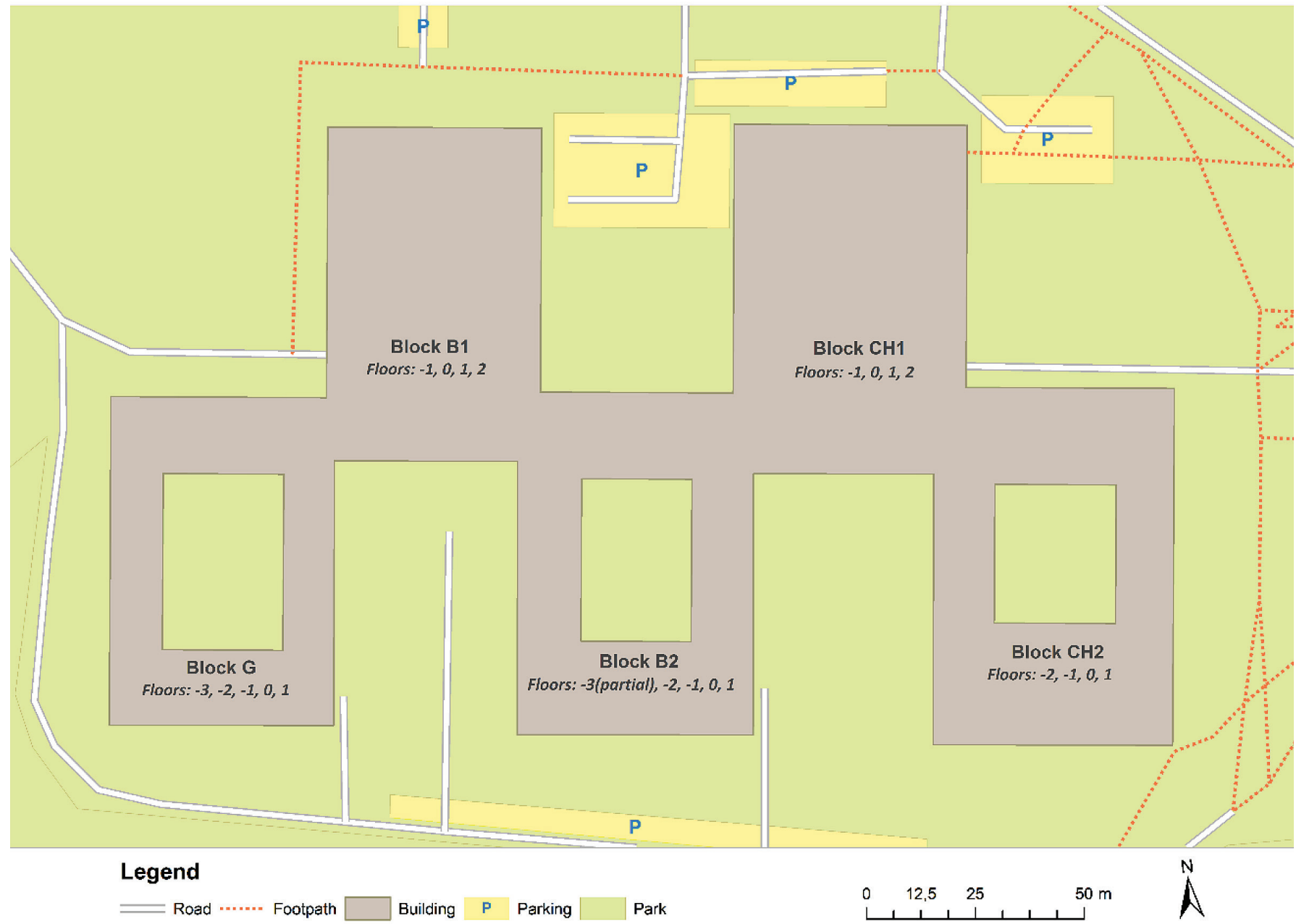

Fig. 2. Building complex of FNS: Five blocks with various amounts of floors (data source: OpenStreetMap, ${ }^{\odot}$ OpenStreetMap (and) contributors, $C C-B Y-S A$ ) 
Since we had not assumed any frequent changes in the source code or in the building's structure, it was possible to leverage Local Storage and Service Worker to create a fully offline-capable application. Such software dramatically decreases the hardware and bandwidth requirements for the server. What is more, the application's startup time, its responsivity, and ability to run without any network connection also promotes accessibility and the user's experience of the entire solution. Finally, with the Web Application Manifest, users can also pin the application to the home screen of any device.

\subsection{Preparation of the Data for Indoor Navigation}

As mentioned earlier, the building complex of FNS (Fig. 2) consists of five blocks. Every block is unique and differs in a few ways: the presence of an atrium, the presence of a lecture hall, various floor ranges, etc. The blocks are connected by a single hallway on each floor that they have in common. In order to create indoor navigation, two data requirements had to be met: the spatial representation of the interior with every potential intersection (e.g., passages, stairs or elevators) and the identification number of each room in the complex. However, FNS does not maintain any relevant digital spatial information for indoor navigation in the building complex, so we had to analyze the available analogue sources provided by the management of FNS to create a digital foundation based on them. The emergency evacuation plans met both requirements, so we decided to scan their analogue forms using the Nashuatec MPC2011 scanner.

While raster scans can serve as a good base layer on a map, a routing algorithm requires input in the form of points and lines expressed as a set of coordinates. Therefore, we had to create an additional two layers based on scans. Although we used the proprietary ArcGIS 10.2.2 to create those layers, such a task can be completed with many other open source or web-based software. The first layer was composed of vertices, including all the breakpoints, intersection points, and POIs, i.e., the graph nodes (Fig. 3). Breakpoints serve to create nodes without any semantics. On the other hand, the intersection points (elevators, staircases) and POIs (rooms, cafeterias, buffets, shops, etc.) have additional significance that are stored as attribute values. The second layer contains path segments between the nodes and represents possible navigation routes, i.e., graph edges. A combination of all the POIs, breakpoints, and line segments represents a suitable graph for Dijkstra's routing algorithm (Fig. 3).

\subsection{Estimation of a Device's Location}

One of the disadvantages of calculating an actual location using the IMS is the fact that the initial location has to be specified. That is necessary, because the plain IMS cannot be used to determine the absolute location; instead, it can just determine the device's motion and its direction. On the other hand, since the majority of the potential use cases rely on the fact that the most probable point of origin is the main entrance, such a requirement is not really limiting. Even if the main entrance is not the point of origin, there is only a small chance of human error because every room in the building complex of FNS is marked with its identification number.

As mentioned in Section 2, we chose to implement the walk detection. In most cases, the principle of this approach is based on monitoring a device's acceleration, specifically its norm (the square root of the sum of the squares of the measured values on all the axes). If the value exceeds the "walk threshold," the application will start the measurement time required to calculate the distance from the initial position. The walk threshold represents the minimal acceleration of

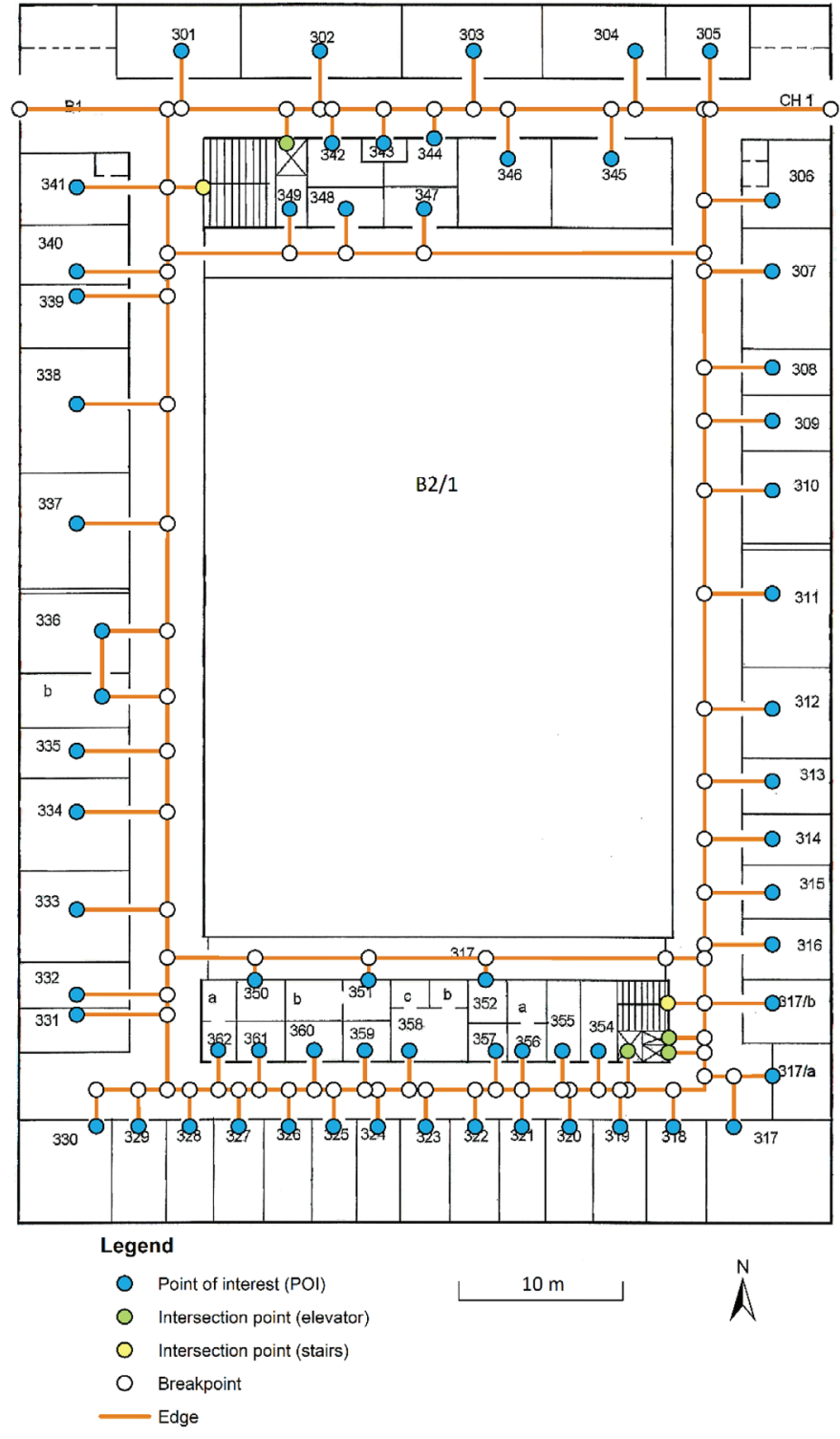

Fig. 3. Sample of graph (nodes and edges) based on emergency evacuation plans of FNS

devices, which are assumed to be a walk. Hence the walk detection strongly relies on the known threshold at the time of navigation. However, the values observed and therefore, the walk threshold can differ among various accelerometer sensors. The chart in Figure 4 presents a comparison of the acceleration between two devices: the Huawei HTC One M7 and the Xiaomi Mi 4i. The test took place at the same time when both devices were placed on the same solid surface and were not moved. Each device recorded acceleration values for exactly ten seconds. There are two interesting facts that can be observed in this comparison. Firstly, the Mi $4 \mathrm{i}$ provides measurements at a higher frequency than the HTC One M7 (approximately 5\% more observations). Secondly, the values observed (maximum, minimum, and average) differ significantly. This test confirms that the walk threshold has to at least be defined individually for each type of device. We proposed to solve this problem with the initial measurement that takes place when the application runs for the first time. In practice the application prompts the user not to walk for a short period of time while it is collecting the acceleration values. The user is encouraged to perform gestures like finger swipes because they produce different accel- 


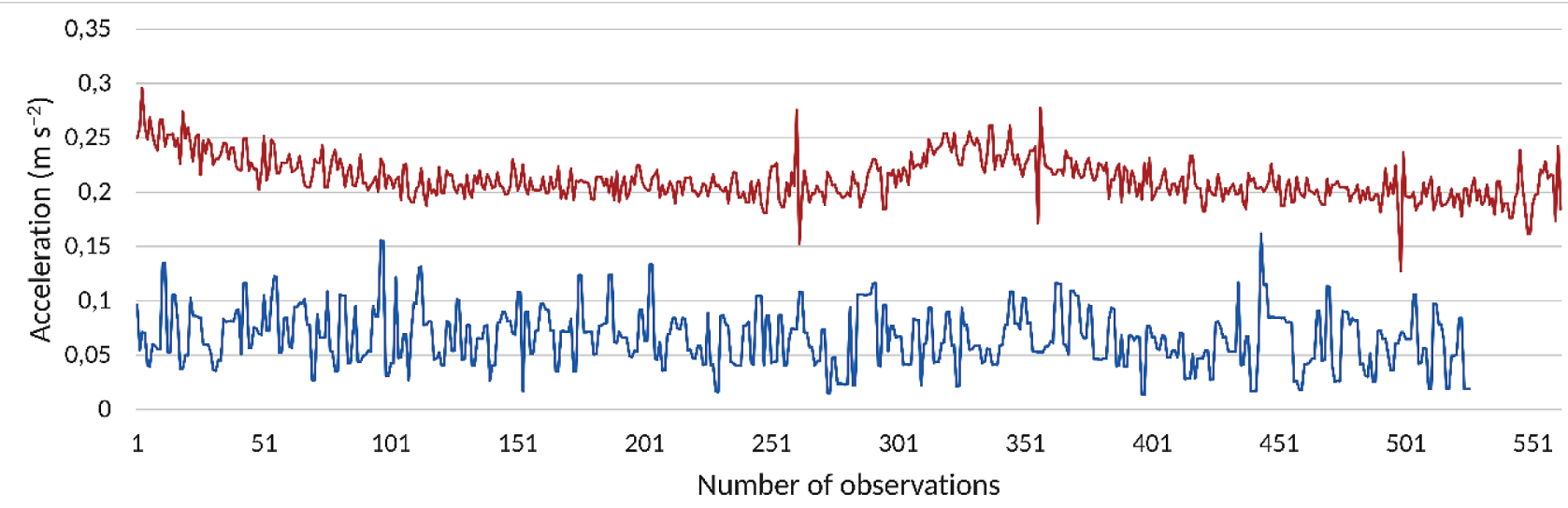

— Huawei HTC One M7 —Xiaomi Mi 4i

Fig. 4. Comparison of acceleration of devices

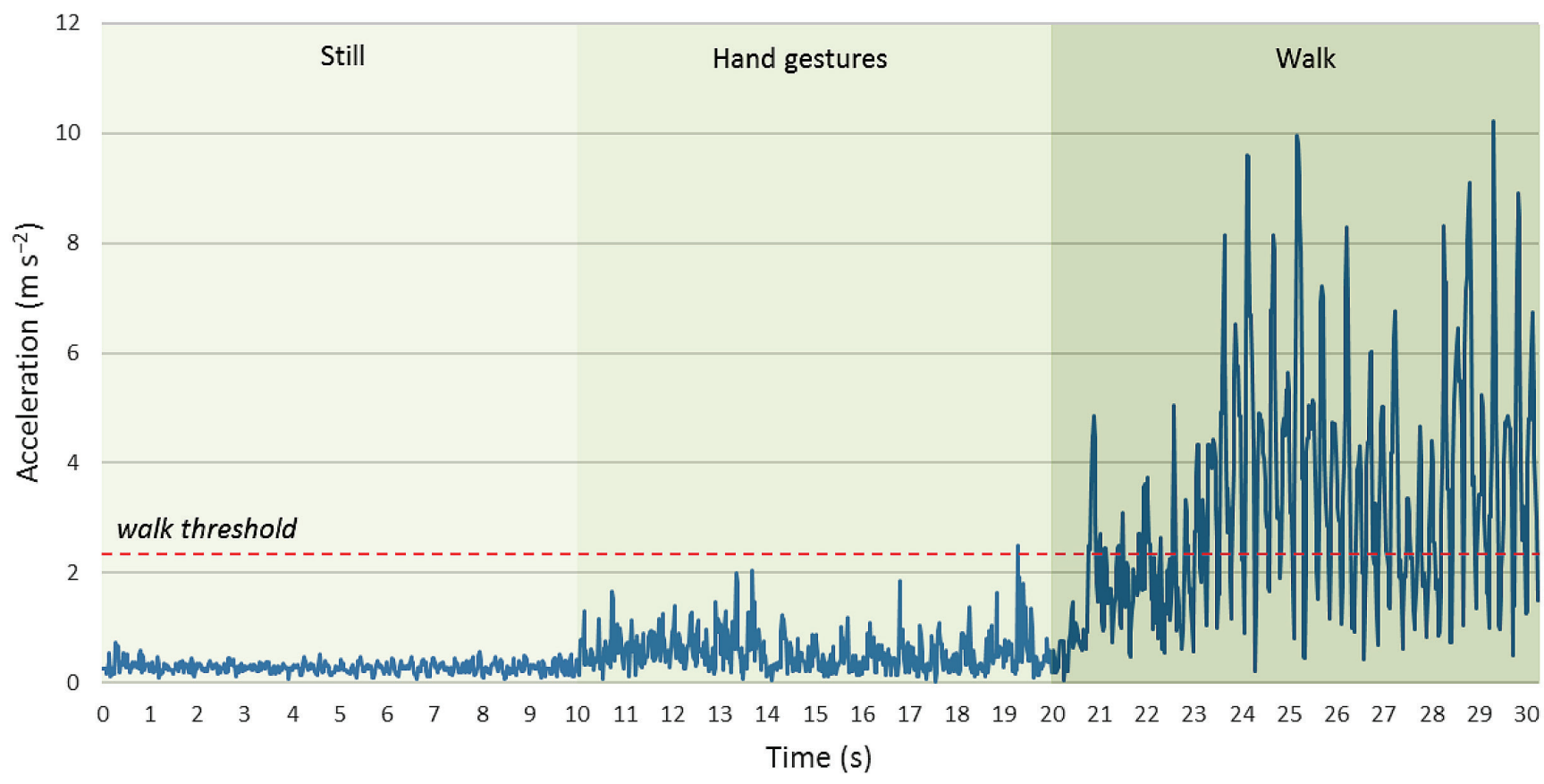

Fig. 5. Acceleration of device over time (Xiaomi Mi 4i)

eration values than if the user holds the device still (Fig. 5). Based on these measurements, the walk threshold is determined as the maximal measured acceleration, and its value is saved to the browser's Local Storage for any subsequent use of the application.

One of the main disadvantages of the walk detection is the fact that the user is considered to be moving while the acceleration values are above the threshold. In the case of the Mi $4 \mathrm{i}$, approximately $39 \%$ of the observation values measured while walking were below the threshold (see the chart in Figure 5). This causes significantly less time to be considered as a walk; therefore, shorter distances have to be calculated.

In theory this could be solved by adjusting the walking speed of an individual, but we resolved this problem with the detection of the delayed stillness. In practice, if the application observes an acceleration value greater than the walk threshold, it will consider the person to be walking and start the step timer. Its purpose is to count down a period of time equivalent to the approximate amount of time required to take a single step. If there is an acceleration value that exceeds the threshold within the period, the application will consider the person to be walking and reset the timer. Otherwise, the person is considered to be still. This method (Fig. 6) allows us to ignore the values that would be otherwise be classified as standing.

If the application detects the walk, it will start measuring the periods of time between each devicemotion event. Therefore, the distance per observation is calculated as the measured time multiplied by the walking speed. According to Neumann (2002), the walking speed of an adult person is roughly $5.15 \mathrm{~km} / \mathrm{h}$, which is also used in our application. The motion vector is then composed from the distance calculated and the last measured azimuth provided by the deviceorientation API. Finally, the application computes the actual location using the motion vector and updates it on the map. This process is repeated approximately 50 times per second (depending on the device).

If a route contains an intersection point, the behavior of the application will slightly change. The application will create a proxim- 


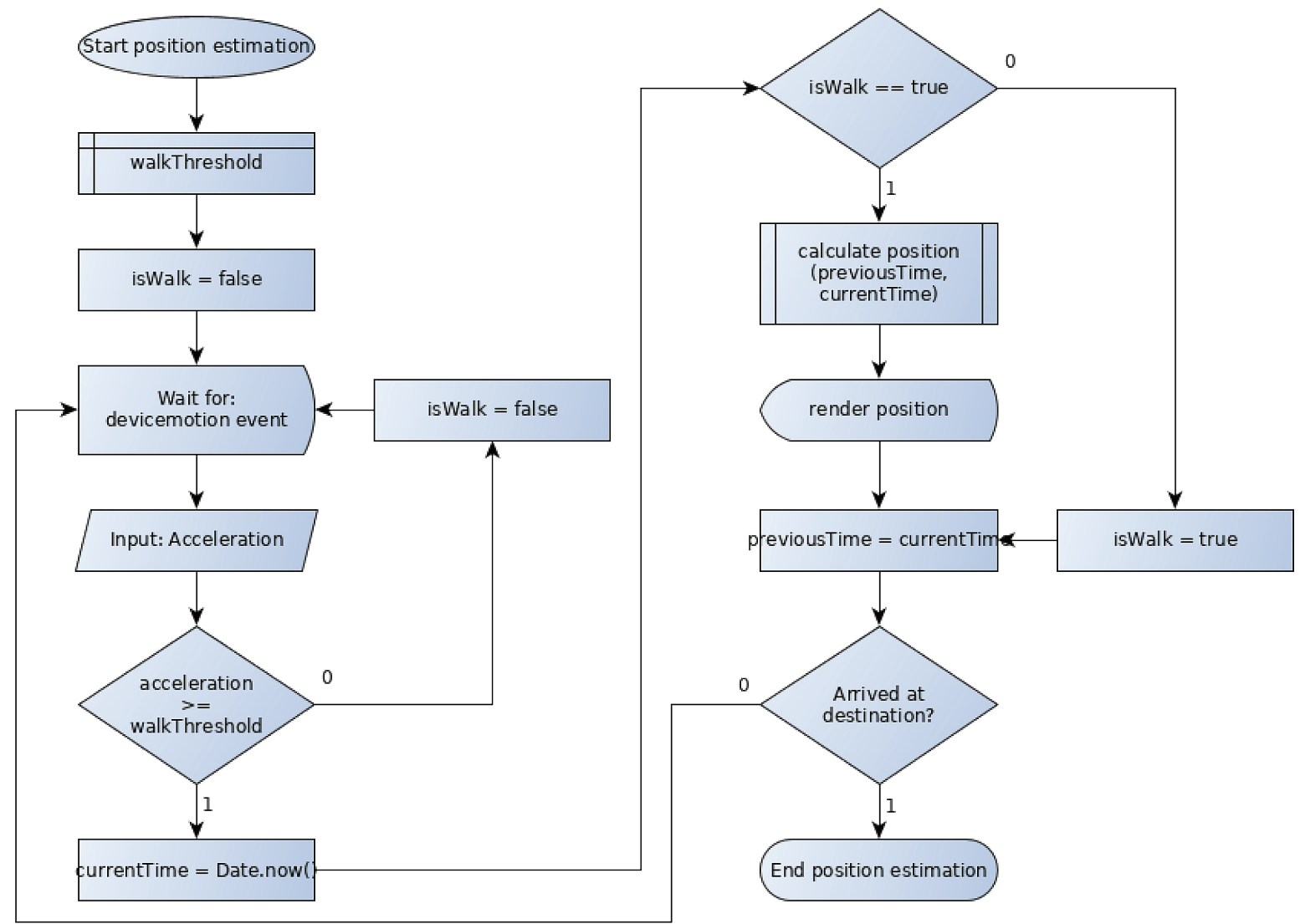

Fig. 6. Estimation of position flowchart diagram

ity buffer around the intersection point. When the device enters the buffer, the position on the map will be snapped to an elevator, while the motion changes become tracked as a background process. If the device overcomes the distance equal to the approximate height of the ceiling ( $3.2 \mathrm{~m}$ in the case of FNS), the subsequent floors are rendered on the map. The application is aware of the amount of floors that the user has to overcome in order to reach the destination and displays them accordingly. When the user reaches the target floor, the application will return to the position of the computation. If the user exits from the elevator prematurely, the application will still show the user's position in the elevator until the destination floor is reached.

While the application should compute the actual location with a sufficient degree of precision, in some cases (e.g., running) the users may experience inaccuracies. In such a scenario, the user always has the ability to change the point of origin to the actual location. All in all, it is advisable to verify the actual location by occasionally checking the numbers of the floors and rooms.

\subsection{Calculation of the Shortest Route}

Before carrying out the network analysis, a graph has to be constructed (Fig. 3). The jKstra library is equipped with capabilities for 2D graph construction as well as built-in routing algorithms. The graph was constructed using the instance of the jkstra.Graph. It is then extended by each vertex using the graph's addVertex method. The vertices (i.e., the graph nodes) store both their spatial and attribute properties in a data record, which contains every piece of information about the node: the coordinates, floor number and an optional special purpose (e.g. it is an intersection point, POI, etc.). The creation of the node is followed by the construction of the edge, which is accomplished with the addEdgePair method. This method creates a bi-directional relation between points with additional records similar to the vertex data containing information about the edges. We used this record to store the distance calculated between the nodes.

The computation of the shortest path is carried out by the instance of jkstra.algos.Dijkstra with the graph supplied. The calculated route segments are obtained by invoking its shortestPath method with the origin and target provided as functional arguments. Finally, the returned segment sequence is transformed into a single line string and supplied to an adequate vector source. While the jKstra library enables routing between any nodes, for obvious reasons our application allows the user only to choose from the POIs.

\subsection{Spatial Data Visualization}

There are three layers that have to be rendered in the application: the base layer, proposed navigation path and actual location of the device. The concept of the layers in the OL library consists of the instances created from the objects inherited from ol.layer.Layer. The object's properties vary among its different layer types, but each must be bound to some kind of data source, either a raster or vector.

The base layer currently consists of multiple emergency evacuation plan scans. To visualize the static images, an instance of ol.layer. Image is created with the source of ol.source.ImageStatic. In order to load the image, it is necessary to provide its uniform resource locator (URL). The fetched image is cached and rendered onto the map. 
Since the scans are only static pictures, it is also necessary to specify the spatial extent of every image on the map coordinates, so it can be georeferenced. The rendering process of multiple high-DPI pictures can be slow, especially on mobile devices. For this reason, we decided to represent the floors as sub-layers: only one floor is rendered at a time.

Both the device's position and path segments are present in the form of the instance of ol.layer.Vector with the source of ol.source. Vector. Obviously, the device's position is represented by the point's geometric type, and the navigation route contains a single line composed of the segments. Since both are computed dynamically, the initial state of the vector sources contains exactly zero geometries. The vector features are pushed into the sources via the addFeature method. While the actual position is just updated using the setGeometry method of ol.Feature, the proposed navigation routes are always created anew. Both layers are rendered with a specific style object, i.e., an instance of ol.style.Style.

\section{RESULTS AND DISCUSSION}

In this paper we have presented a simple, yet capable, indoor navigation system designed from scratch using only analogue emergency evacuation plans. In contrast to the previous approaches, we focused on simplicity and accessibility. The flow chart of the proposed webbased indoor navigation system is shown in Figure 7. The resulting map with the rendered emergency evacuation plans, actual position, and calculated navigation route is shown in (Fig. 8).

The application is built on public web standards and open source libraries and relies on the hardware components found in the majority of smartphones. The ability to navigate without a network connection reduces the computation and bandwidth requirements for the server only to the initial page loading. Furthermore, the application does not require any complex data input, nor does it need any significant data pre-processing due to its ability to use a simple routing algorithm. It allows the user to specify the POIs, based on which it calculates and displays the shortest route. To determine the approximate location, we leveraged the fact that most of currently used smartphones provide an API for the device's motion and orientation. To provide a more accurate determination of a position, we proposed and used a modified walk detection method as well as the initial calibration of the device's motion.

While the application requires more extensive testing using broader range of hand-held devices (both smartphones and tablets) as well as testing by various testers (walk speed of an individual impacts spatial precision), the initial testing using a mobile Apple iPhone yielded sufficient accuracy over short-to-medium distances. Since the precision decreases over distance, users are also advised to verify

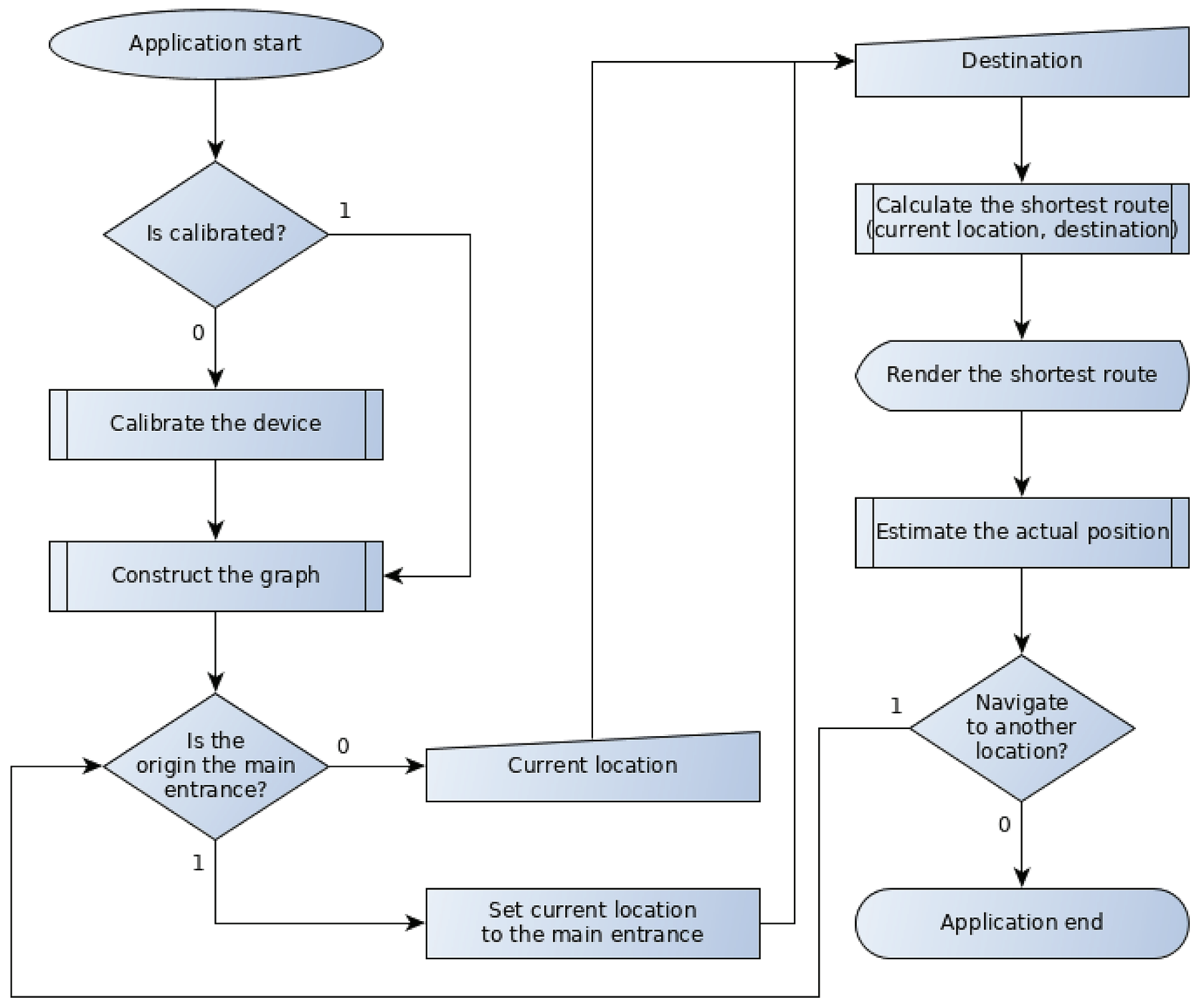

Fig. 7. Flow chart of the proposed web-based indoor navigation system 


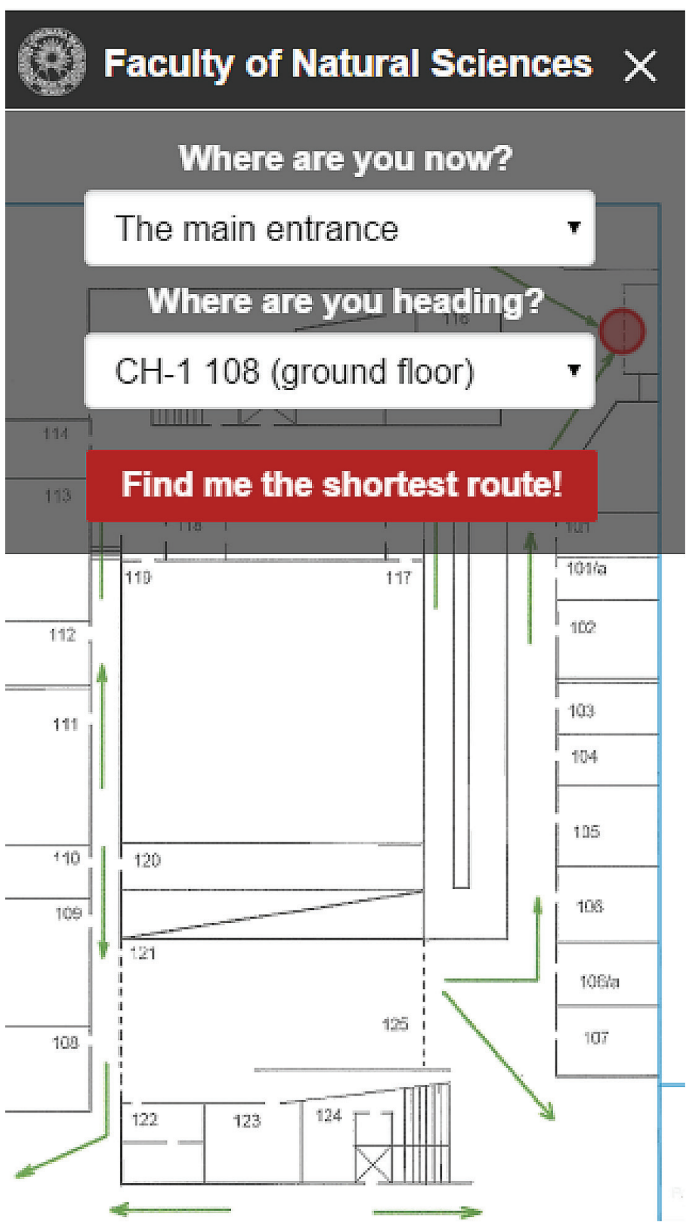

\section{Faculty of Natural Sciences $=$}

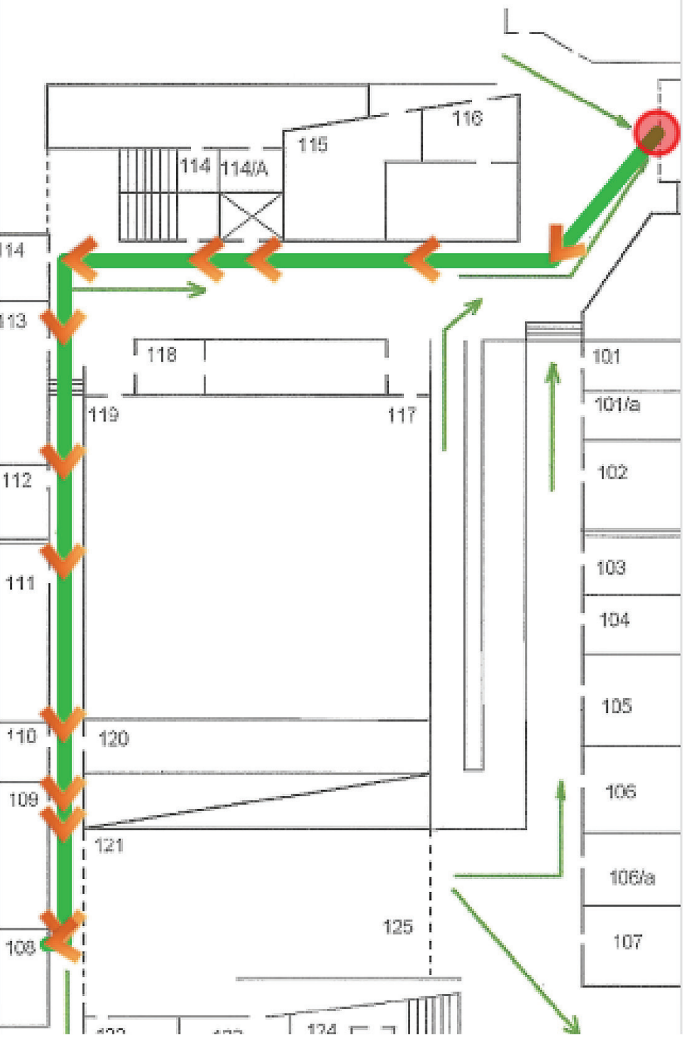

Fig. 8. User interface of the web-based indoor navigation system implemented

their position from time to time using room numbers, POIs, etc. This also applies to horizontal movement such as moving between floors using both elevators and stairways.

Even though the application possesses the core functionality of a navigation system, we suggest several possible improvements. Firstly, we recommend using Wi-Fi triangulation as an auxiliary technology to increase the accuracy of the user's actual location. At the time of writing this article, there was no browser with API capable of delivering the crucial signal data, but there are efforts to define the Network Information API (https://dvcs.w3.org/hg/dap/raw-file/ tip/network-api/Overview.html). Google's Chrome Canary already supports some of its features, though just in an experimental mode. We believe that such an API could be extended to provide the data necessary for triangulation. Secondly, the transition between floors can be refined with a double integration of the acceleration. While this technique becomes less accurate over time, it could be ideal for the detection of vertical motion in an elevator. In the future, an application could also rely on barometric measurements to detect vertical movement on staircases as described by Jeon (2015). It was not possible to implement this method due to the lack of a browser API. On the other hand, barometric sensors are increasingly becoming standard equipment of smartphones, and it is possible that such an API will be soon available. QR codes might serve as the supplementary means for determination of a user's original location as well as to provide additional information about the site. Unlike the previously suggested improvements, it is possible to read QR codes in a web-based application today. Last, but not least, the data visualization could be improved by the use of better base maps than the evacuation plans.
Furthermore, 3D floor visualization would provide a much better user experience. Our design enables such an extension, because the OL library is capable of 3D rendering in conjunction with the Cesium library (https://cesiumjs.org/). While 3D visualization is indeed user-friendly, it also requires notable investment in its development as well as data processing. Obviously, improving the system will also increase the cost of its implementation. However, even in its present form it meets the essential quality requirements for an indoor navigation system and is immediately suitable for use.

\section{CONCLUSIONS}

In this paper, we have proposed an architecture for an application that does not require any significant investment in software development or infrastructural construction. The resulting application represents fully-featured indoor navigation software. It is independent of any external hardware or operating system while remaining mobile-friendly. In spite of its lower degree of precision and the need to specify both the origin and destination, the navigation has been successfully tested in several scenarios inside the building complex of FNS. For this reason, we believe that our application could potentially be used in other similar structures, where the people often get disoriented or even lost.

\section{ACKNOWLEDGEMENT}

This work was supported by Grant No. 1/0682/16 of the VEGA Grant Agency of the Slovak Republic. 


\section{REFERENCES}

Choo, J. H. - Cheong, S. N. - Lee, Y. L. (2014) Design and Development of NFC Smartphone Indoor Interactive Navigation System. World Applied Sciences Journal, Vol. 29, No. 6, 738-742 pp. DOI: 10.5829/idosi.wasj.2014.29.06.1553

Costa-Montenegro, E. - Gonzalez-Castano, F. J. - Conde-Lagoa, D. - Barragans-Martinez, A. B. - Rodriguez-Hernandez, P. S. - Gil-Castineira, F. (2011) QRMaps: an efficient tool for indoor user location based on $Q R$ codes and Google maps. 2011 IEEE Consumer Communications and Networking Conference (CCNC), IEEE, LasVegas, 928-932 pp. DOI: 10.1109/ CCNC.2011.5766643

Dardari, D. - Closas, P. - Djurić, P. M. (2015) Indoor Tracking: Theory, Methods, and Technologies. IEEE Transactions on Vehicular Technology, Vol. 64, No. 4, pp. 1263-1278. DOI: 10.1109/ TVT.2015.2403868

Dijkstra, E. W. (1959) A note on two problems in connection with graphs. Numerische Mathematik, No. 1, pp. 269-271.

Geng, L. - Bugallo, M. F. - Athalye, A. - Djurić, P. M. (2014) Indoor Tracking With RFID Systems. IEEE Journal of Selected Topics in Signal Processing, Vol. 8, No. 1, pp. 96-105. DOI: 10.1109/ JSTSP.2013.2286972

Groves, D. (2008) Principles of GNSS, Inertial and Multisensor Navigation Systems. UK: Artech House, London, 536 pp.

Gu, Y. - Lo, A. - Niemegeers, I. (2009) A Survey of Indoor Positioning Systems for Wireless Personal Networks. IEEE Communications Surveys and Tutorials, No. 11, pp. 13-32. DOI: 10.1109/ SURV.2009.090103.

Gualda, D. - Ureña, J. - García, J. - Lindo, A. (2014) Locally-referenced ultrasonic - LPS for localization and navigation. Sensors, Vol. 14, No. 11, pp. 21750-21769.

Hazas, M. - Hopper, A. (2006) Broadband ultrasonic location systems for improved indoor positioning. IEEE Transactions on Mobile Computing, Vol. 5, No. 5, pp. 536-547. DOI: 10.1109/ TMC.2006.57

Hofmann-Wellenhof, B. - Lichtenegger, H. - Collins, J. (2001) Global Positioning System, Theory and Practice. Springer Verlag, Vienna, Austria, 389 pp.

Ijaz, F. - Yang, H. K. - Ahmad, A. W. (2013) Indoor Positioning: A Review of Indoor Ultrasonic Positioning Systems. Advanced Communication Technology (ICACT), $15^{\text {th }}$ International Conference, IEEE, PyeongChang, South Korea, pp. 1146-1150.

Ilkovičová, L. - Kajánek, P. - Kopáčik, A. (2016) Pedestrian Indoor Positioning and Tracking using Smartphone Sensors, Step Detection and Map Matching Algorithm. Geodetski list, Vol. 70 (93), No. 1, pp. 1-24.

Jain, M. - Rahul, R. C. P. - Tolety, S. B. (2013) A study on indoor navigation techniques using smartphones. Advances in Computing, Communications and Informatics (ICACCI): IEEE Conference Publications, IEEE, Jaipur, India, pp. 1113-1118 DOI: 10.1109/ICACCI.2013.6637333

Jeon, J. - Kong, Y. - Kangbin, Y. - Nam, Y. - Yim, K. (2015) An Indoor Positioning System using Bluetooth RSSI with an Accelerometer and a Barometer on a Smartphone. $201510^{\text {th }}$ International
Conference on Broadband and Wireless Computing, Communication and Applications: (BWCCA): IEEE Conference Publications, pp. 528-531. DOI: 10.1109/BWCCA.2015.142

Koyuncu, H. - Yang, S. H. (2010) A Survey of Indoor Positioning and Object Locating Systems. International Journal of Computer Science and Network Security, Vol. 10, No. 5. pp. 121-128.

Lim, J. - Lee, S. J. - Tewolde, G. - Kwon, J. (2014) Ultrasonic-sensor deployment strategies and use of smartphone sensors for mobile robot navigation in indoor environment. 2014 IEEE International Conference on Electro/Information Technology, WI: IEEE, Milwaukee, pp. 410-415, DOI: 10.1109/EIT.2014.6871831

Le, M. H. V. - Saragas, D. - Webb, N. M. - Wyglinski, A. M. - Vaz, R. F. (2009) Indoor Navigation System for Handheld Devices. A Major Qualifying Project Report. Worcester, Worcester Polytechnic Institute, UK.

Leick, A. (2004) GPS Satellite Surveying. John Wiley \& Sons, Hoboken, NJ, 435 pp.

Ma, R. - Guo, Q. - Hu, Ch. - Xue, J. (2015) An Improved WiFi Indoor Positioning Algorithm by Weighted Fusion. Sensors, Vol. 15, No. 9, pp. 21824-21843. DOI: 10.3390/s150921824

Mautz, R. (2009) Overview of Current Indoor Positioning Systems. Geodezija ir Kartografija. Vol. 35, No. 1, pp. 18-22. DOI: 10.3846/1392-1541.2009.35.18-22

Mautz, R. (2012) Indoor Positioning Technologies. (Unpublished habilitation thesis). Zurich, Swiss Federal Institute of Technology in Zurich, Switzerland.

Neumann, D. (2002) Kinesiology of the musculoskeletal system: foundation for physical rehabilitation. MO: Mosby Elsevier, St. Louis, $597 \mathrm{pp}$

Ozdenizci, B. - Coskun, V. - Ok, K. (2015) NFC Internal: An Indoor Navigation System. Sensors, Vol. 15, No. 4, pp. 7571-7595. DOI: $10.3390 / \mathrm{s} 150407571$

Rodriguez, A. - Prieto, I. - Montanés, J. (2013) Smart indoor positioning/location and navigation. International Journal of Artificial Intelligence and Interactive Multimedia, Vol. 2, No. 2, pp. 43-50. DOI: 10.9781/ijimai.2013.225

Shandilya, S. - Idate, S. R. (2015) Wi-Fi Assistant Triangulation and Navigation System for an Indoor Environment. Advances in Computer Science and Information Technology, Vol. 2, No. 10, pp. 95-99.

Woodman, O. J. (2007) An introduction to inertial navigation. Technical report 696. Cambridge, University of Cambridge, UK.

Yasir, M. - Ho, S. W. - Vellambi B. N. (2016) Indoor position tracking using multiple optical receivers. Journal of Lightwave Technology, Vol. 34, No. 4, pp. 1166-1176. DOI: 10.1109/ JLT.2015.2507182

Zeng, W. - Church, R. L. (2009) Finding shortest paths on real road networks: the case for $A^{*}$. International Journal of Geographical Information Science, Vol. 23, No. 4, pp. 531-543. DOI: $10.1080 / 13658810801949850$ 